\title{
Corpus callosum index correlates with brain volumetry and disability in multiple sclerosis patients
}

Stefanus E. Sugijono, MD, Rahmad Mulyadi, MD, Salsabila Firdausia, MD, Joedo Prihartono, MD, Riwanti Estiasari, MD.

\section{ABSTRACT}

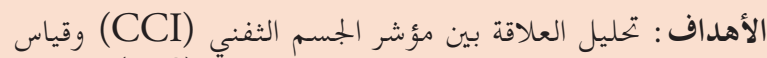

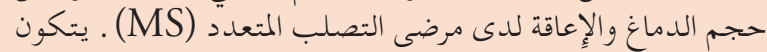

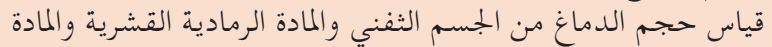
الرمادية تحت القشرية وأحجام المادة البيضاء.

المنهجية : أجريت هذه دراسة مستعرضة بأثر رجعي خلال الفترة

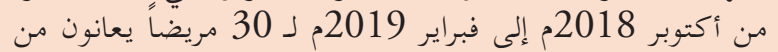

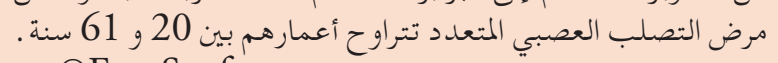

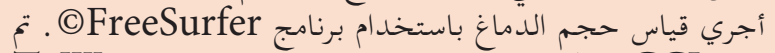

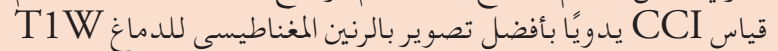

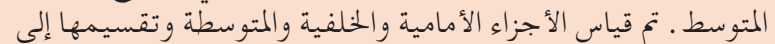

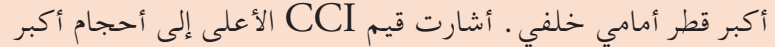

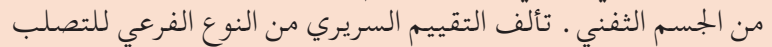

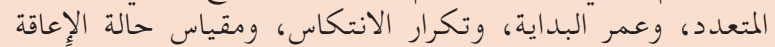

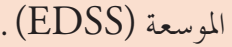

النتائج : اشتملت الدراسة على 30 مرضى MS النقام مع مع متوسط العمر

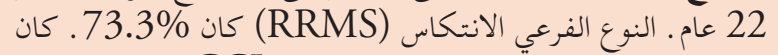

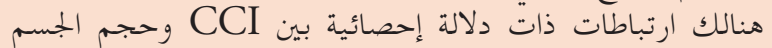

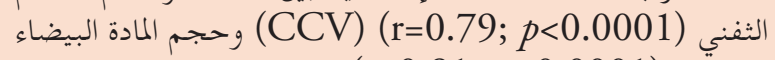
الدماغية (r=0.81; $p<0.0001$ (r=0) ) ظهرت ارتباطات كبيرة بين وحجم المادة الرمادية القشرية (r=0.64;

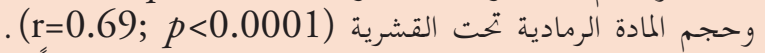

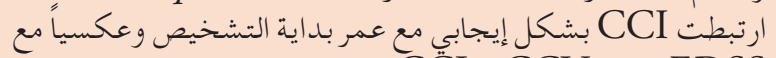

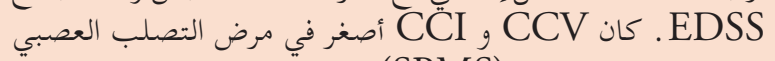
المتعدد الثانوي الثانوي (SPMS) .

الخلاصة : إن CCI سهل وسريع الحصول عليه في التصوير بالرنين

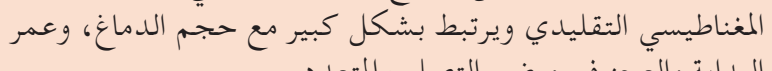

Objectives: To analyze the correlation between corpus callosum index (CCI), brain volumetry, and disability in multiple sclerosis (MS) patients. The brain volumetry consists of the corpus callosum, cortical gray matter, subcortical gray matter, and white matter volumes.
Methods: This was a retrospective cross-sectional study from October 2018 to February 2019 of 30 patients with MS aged 20 to 61 years old. Brain volumetry was performed using FreeSurfer@ software. The CCI were measured manually using conventional best mid-sagittal T1W brain MRI. The anterior, posterior, and medium segments were measured and divided to its greatest anteroposterior diameter. Higher CCI values indicated greater corpus callosum volumes. Clinical evaluation was comprised of MS subtype, age of onset, relapse frequency and Expanded Disability Status Scale (EDSS).

Results: Thirty MS patients with median of age 22 years were included. Relapsing-remitting (RRMS) subtype were $73.3 \%$. Very significant correlations were shown between the CCI and corpus callosum volume $(\mathrm{CCV}) \quad(\mathrm{r}=0.79 ; p<0.0001)$ and cerebral white matter volume $(\mathrm{r}=0.81 ; p<0.0001)$. Significant correlations were shown between the $\mathrm{CCI}$ and cortical gray matter volume $(\mathrm{r}=0.64 ; p<0.0001)$ and subcortical gray matter volume $(\mathrm{r}=0.69 ; p<0.0001)$. The CCI was positively correlated with age of onset and inversely with EDSS. The CCV and CCI were smaller in secondary progressive MS (SPMS).

Conclusion: The CCI is easy and fast to obtain in conventional MRI and significantly correlated with brain volumetry, age of onset and disability in MS patients.

Neurosciences 2020; Vol. 25 (3): 193-199 doi: 10.17712/nsj.2020.3.20190093

From the Department of Radiology (Sugijono), Division of Neuroradiology (Mulyadi), Department of Radiology, Department of Neurology (Firdausia, Estiasari), Department of Community Medicine (Prihartono), Faculty of Medicine, University of Indonesia, Cipto Mangunkusumo Hospital, Jakarta, Indonesia.

Received 6th October 2019. Accepted 15th April 2020.

Address correspondence and reprint request to: Dr. Rahmad Mulyadi, Division of Neuroradiology, Department of Radiology, Faculty of Medicine, University of Indonesia, Cipto Mangunkusumo Hospital, Jakarta,Indonesia.E-mail:dr_rahmad_radiologi@yahoo.com ORCID ID: https://orcid.org/0000-0002-0246-6088 
$\mathrm{M}$ ultiple sclerosis (MS) is one of the most common neurological diseases of the central nervous system and has various clinical manifestations, affecting the sensory, motor, cerebellar, brainstem, and autonomic systems. ${ }^{1-4}$ The MS progression will lead to disability that can affect the quality of life. In 2013, the prevalence of MS was 33 per 100,000 globally, an increase from approximately 30 per 100,000 in $2008 . .^{5}$ Regarding the cognitive impairment, it has been found in a study by Rao et $\mathrm{al}^{6}$ a $45 \%$ frequency of cognitive impairment in MS patients. Furthermore, it has been found in several studies that the decline in visual and verbal episodic memory as well as decelerated cognitive processing speed are the most frequent cognitive domains impaired in multiple sclerosis. ${ }^{7}$ Between these 2 domains, it has been shown that memory impairment were slightly more common than those with memory impairment and processing speed impairment in 128 patients with relapsing-remitting MS (RRMS). ${ }^{8}$ In our previous study, we found impairments in the Symbol Digit Modality test (up to 50\%), California Verbal Learning test-II (27.5\%), and Brief Visuospatial Memory test-revised (32.5\%) in MS patients. ${ }^{9}$

Brain volume in MS patients were found to be significantly smaller compared to healthy subjects and associated with the progression of disability. ${ }^{10}$ Brain atrophy in MS can occur by 3 mechanisms: volume loss within the lesion itself, retrograde degeneration, and Wallerian degeneration in the remote area of the fiber pathway. ${ }^{4,11,12}$ Brain atrophy can be seen in earliest stages of MS (clinically isolated syndrome)..$^{13}$ Gray matter atrophy begins early in the course of the disease and is correlated with the progression of disability, especially motor and cognitive disability. ${ }^{14-16}$ Measuring brain atrophy has been proposed as one of several treatment monitoring parameters for MS. ${ }^{17}$

The corpus callosum is one of the main white matter pathways and affected by the progress of chronic diseases, including MS. ${ }^{1,3,4}$ Corpus callosum damage is correlated with cognitive impairment and motor disability in MS patients. Corpus callosum atrophy could be a clinically relevant marker of cognitive impairment. ${ }^{18-20}$

Brain volumetry using magnetic resonance imaging

Disclosure. Authors have no conflict of interests, and the work was not supported or funded by any drug company. This study was approved by Health Research Ethics Committee Faculty of Medicine (FM), University of Indonesia (UI) (No. 90/UN2.F1/ETIK/2019).
(MRI) is a useful, noninvasive tool in assessing subcortical morphometric changes as well as evaluating the regional neurological impact of psychopathology, such as dementia, psychiatric disorders, and normal aging. ${ }^{21}$ Some software packages have been developed for measuring brain tissue volume using MRI with semi-automatic segmentation, such as FreeSurfer(C) (The General Hospital Corporation, Boston MA, USA), FIRST (FMRIB's Integrated Registration and Segmentation Tool), FSL (FMRIB's Software Library), and SPM (Statistical Parametric Mapping). ${ }^{22}$

With this background, the objective of this study was to determine the correlation between the corpus callosum index (CCI) measurements and the corpus callosum, cortical gray matter, subcortical gray, and cerebral white matter volumes determined through brain MRI volumetry and clinical characteristics in MS patients.

Methods. This was a retrospective cross-sectional study that was conducted using the medical record and MRI data, collected from October 2018 to February 2019, of 30 MS patients. The patients that were included in this study: (1) fulfilled the McDonald criteria of 2010; (2) were 18-65 years of age; and (3) gave their written consent to participate in the study. Patients with the following criteria were excluded: (1) patients whose MRIs included motion artifacts that affected the CCI measurement, and (2) patients with comorbid brain disease that affected brain tissue volume. Institutional review board approvals were obtained for this study from the Health Research Ethics Committee Faculty of Medicine, University of Indonesia - Cipto Mangunkusumo National General Hospital (No. 90/ UN2.F1/ETIK/2019).

Demographic and clinical data (MS subtype, age of onset, frequency of relapse and Extended Disability Status Scale-EDSS) were obtained from the medical records and patient registry. The MRI data were collected from a picture archiving and communication system (PACS) of the Radiology Department of Dr. Cipto Mangunkusumo General Hospital (RSCM) from MS patients. The EDSS data used in this study was the one that was taken not more than 2 weeks from the MRI examination. Only patients with complete clinical and MRI data using below protocols were recruited.

All patients had the same MRI scanning protocol and were acquired on the same scan. Participants were scanned using a $1.5 \mathrm{~T}$ GE Optima MR450w scanner (GE Healthcare, Waukesha, WI, USA). The MRI protocol included T1 SE (TR 475, TE min. full, freq. FOV $22 \mathrm{~cm}$, phase FOV $0.8 \mathrm{~cm}$, slice thickness $5 \mathrm{~mm}$, 
29 slices, bandwidth $31.25 \mathrm{~Hz}$ ), FSPGR BRAVO (TR $10.5 \mathrm{~ms}$, TE $4.3 \mathrm{~ms}$, freq. FOV $22 \mathrm{~cm}$, phase FOV 0.8 $\mathrm{cm}$, slice thickness $1.5 \mathrm{~mm}$, flip angle $11^{\circ}$, bandwidth $20.83 \mathrm{~Hz}$ ), FSPGR BRAVO with contrast (TR 10.5 $\mathrm{ms}$, TE $4.3 \mathrm{~ms}$, freq. POV $22 \mathrm{~cm}$, phase FOV $0.8 \mathrm{~cm}$, slice thickness $1.5 \mathrm{~mm}$, flip angle $11^{\circ}$, bandwidth 20.83 $\mathrm{Hz}$ ), FSPGR-IR 3D (TR 9.2, TE min. full, freq. FOV $22 \mathrm{~cm}$, phase FOV $0.8 \mathrm{~cm}$, slice thickness $1.5 \mathrm{~mm}$, flip angle 10, bandwidth $15.63 \mathrm{~Hz}$ ), T2* GRE (auto TR 660, TE 15, freq. FOV $22 \mathrm{~cm}$, phase FOV 0.75 $\mathrm{cm}$, slice thickness $5 \mathrm{~mm}$, flip angle $20^{\circ}, 29$ slices, bandwidth $14.71 \mathrm{~Hz}$ ), DWI (TR 6000, TE minimum, freq. PV $24 \mathrm{~cm}$, phase POV $1 \mathrm{~cm}$, slice thickness $5 \mathrm{~mm}$, 23 slices, bandwidth $250 \mathrm{~Hz}$ ), and sagittal T2 FLAIR (TR 8000, TE 95 freq. FOV $24 \mathrm{~cm}$, phase FOV 0.8 $\mathrm{cm}$, slice thickness $5 \mathrm{~mm}, 23$ slices, bandwidth 31.25 $\mathrm{Hz}$, echo train length 14).

All the secondary brain volumetry data were

Table 1 - Clinical Characteristics, brain volumetry, and corpus callosum index measurement

\begin{tabular}{lc}
\hline Variables & $\mathrm{N}=30$ \\
\hline Clinical Characteristics & \\
Gender $-N(\%)$ & $6(20)$ \\
Male & $24(80)$ \\
Female & \\
MS subtype - N(\%) & $22(73.3)$ \\
RRMS & $8(26.7)$ \\
SPMS & $32(20-61)$ \\
Age (years) - Median (range) & $26(15-52)$ \\
Age of onset (years) - Median (range) & $4.5(1-12)$ \\
Relapse frequency (times/year) - Median (range) & $3.5(0-8)$ \\
EDSS - Median (range) & \\
Brain volumetry (mm ${ }^{3}$ ) and CCI & $2233.6(696.3)$ \\
Corpus Callosum volume - Mean (SD) & $379396.8(42044.9)$ \\
Cortical gray matter volume - Mean (SD) & $44717.7(6818.8)$ \\
Subcortical gray matter volume - Mean (SD) & $354678.8(66998.5)$ \\
White matter volume -Mean (SD) & $0.31(0.07)$ \\
Corpus Callosum Index - Mean (SD) & \\
\hline
\end{tabular}

Table 2 - The SPMS subjects had higher EDSS and smaller CCV and CCI.

\begin{tabular}{lccc}
\hline Variables & $\begin{array}{c}\text { RRMS (N=22) } \\
\text { Median (range) }\end{array}$ & $\begin{array}{c}\text { SPMS (N=8) } \\
\text { Median (range) }\end{array}$ & ${ }^{*} p$-value \\
\hline Age of onset & $29.5(17-52)$ & $23.5(15-28)$ & 0.06 \\
EDSS & $3(0-7)$ & $6(3.5-8)$ & 0.003 \\
CCV & $2459.35(1446.3-2306.1)$ & $1729(617.8-1924)$ & 0.0004 \\
CCI & $0.33(0.19-0.44)$ & $0.24(0.21-0.28)$ & 0.008 \\
\hline \multicolumn{4}{c}{${ }^{*}$ Mann Whitney U test, EDSS - Extended Disability Status Scale, } \\
CCV - Corpus Callosum Volume, CCI - Corpus Callosum Index, \\
RRMS - Relapsing Remitting Multiple Sclerosis, SPMS - Secondary \\
\multicolumn{4}{r}{ Progressive Multiple Sclerosis } \\
\hline
\end{tabular}

examined by a semi-automatic segmentation method using FreeSurfer(C) software version 6.0.0 (https:// surfer.nmr.mgh.harvard.edu/, Harvard University, Boston, MA, USA) and the data analysis was carried out using a desktop computer with a Linux CentOS system (version 7.5.1804). FreeSurfer processes and analyzes MR images and visualizes the structures and functions of the human brain in several steps, namely the separation of the brain from the skull, image recognition, subcortical segmentation, reconstruction of the cortical surface, cortical segmentation, estimation of the cortical thickness, and finally the longitudinal process in which FreeSurfer measured the volume and thickness of the whole brain and fixing the errors in volume measurement. The image reconstruction occurs within the range of $6-40$ hours. The final result is a table consisting of the whole brain, gray matter, and white matter volumes. ${ }^{25}$

We obtained the CCI using PACS INFINITT software with the method of Figueira. ${ }^{23}$ The CCI was measured on a best mid-sagittal T1WI. We drew a line at the longest anteroposterior diameter of the corpus callosum and a perpendicular at its midline (Figure 1). The anterior ( $\left.\mathrm{aa}^{\prime}\right)$, posterior ( $\left.\mathrm{bb}^{\prime}\right)$, and medium ( $\left.\mathrm{cc}^{\prime}\right)$ segments of the corpus callosum were measured and divided to its greatest anteroposterior diameter (ab). Higher CCI values indicated greater corpus callosum volumes. ${ }^{23}$ Two radiologists (ES and RM) who were blinded to the volumetry results and clinical data measured the CCI together. It took 18 seconds to measure the CCI of each patient.

Statistical analysis. We performed statistical analysis using SPSS 20.0 (SPSS Inc., Chicago IL, USA) and

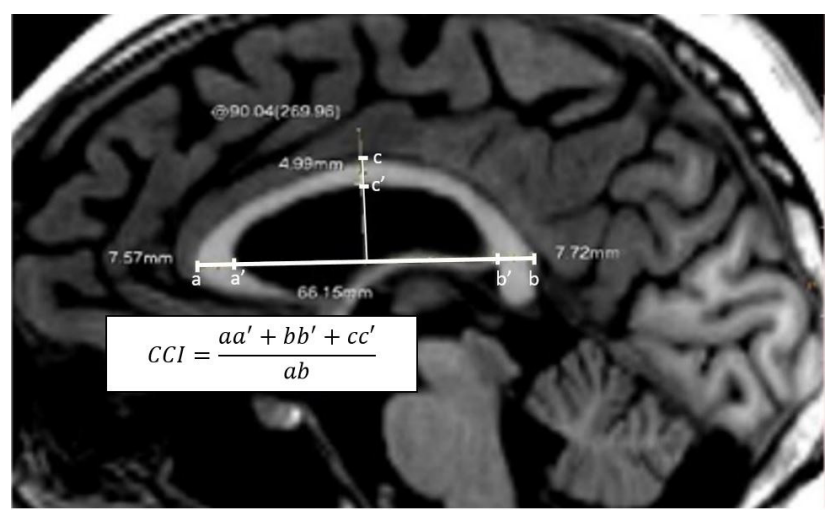

Figure 1- Measurement of CCI using method proposed by Figuera et al. The anterior segment is represented by aa', the posterior by bb' and cc' corresponds to the medium segment. The CC was acquired by normalizing the 3 segments by the farthest anteroposterior segment (ab). 
A
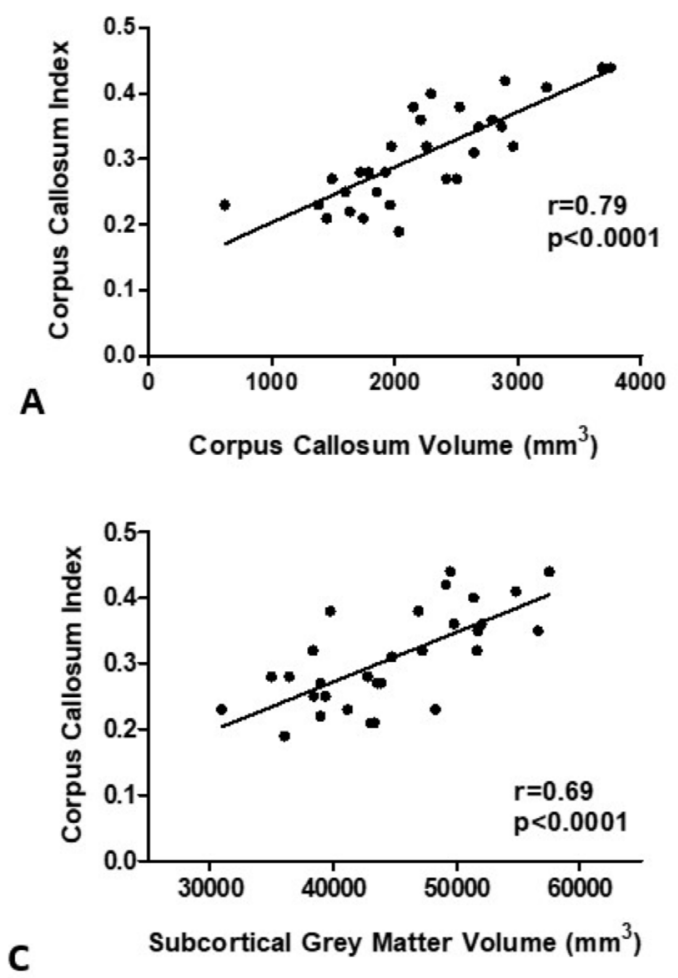
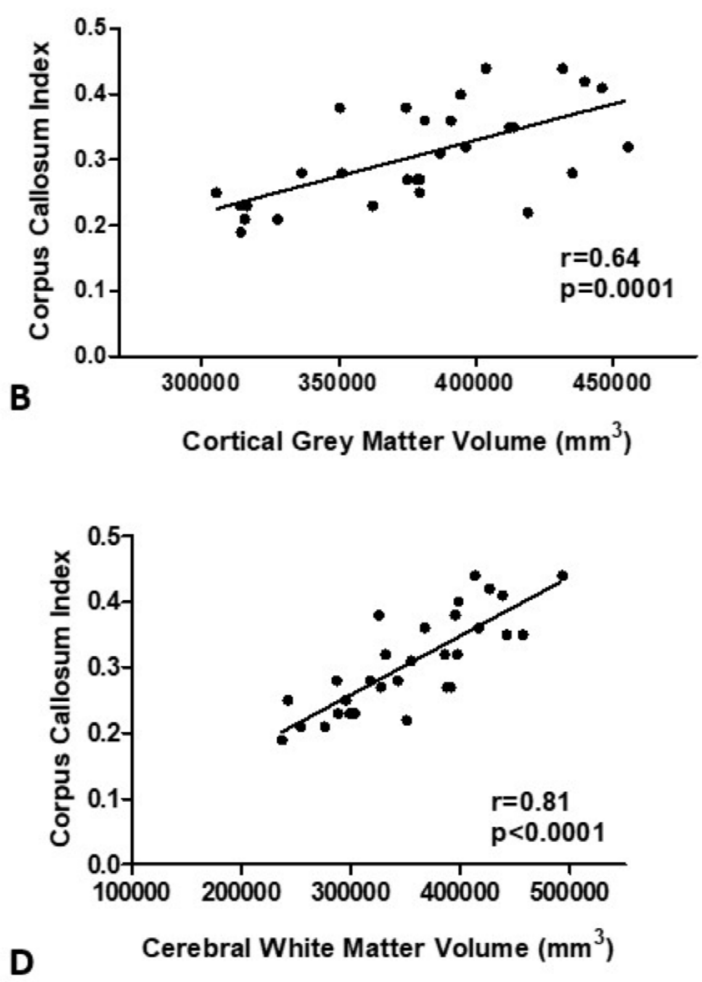

Figure 2 - Correlation between CCI with A) Corpus Callosum volume, B) Cortical Grey Matter volume, C) Subcortical Grey Matter Volume and D) Cerebral White Matter volume

GraphPad Prism 5.0 software (GraphPad Software Inc, La Jolla, Ca). Brain volumetry and CCI measurement results were normally distributed and presented as mean (standard deviation). Clinical data including age, age of onset, EDSS and relapse frequency were not normally distributed and presented as median (range).

Correlation between CCI and corpus callosum, cortical and subcortical grey matter as well as white matter volume were analyzed using Pearson correlation. For the not normally distributed data, we used Spearman correlation. The significance of the correlation was determined by the $r$ value, assessed as follows: $r<0.3$ showed a very non-significant correlation, $r=0.3-0.5$ a non-significant correlation, $\mathrm{r}=0.5-0.7$ a significant correlation, and $\mathrm{r}=0.7-0.9$ a very significant correlation. The different clinical data between RRMS and secondary progressive multiple sclerosis (SPMS) group were analyzed using Mann Whitney U test. $p<0.05$ were considered statistically significant.

Results. Study participants. A total $30 \mathrm{MS}$ patients were included in this study. The male-female ratio was $1: 4$ and $80 \%$ were age below 40 years old. The mean
EDSS was higher in younger ( $\leq 40$ years) compare to older subjects ( 4.1 vs 2.5 ). The youngest onset was 15 and the oldest was 52 years. Majority of the subjects were in RRMS subtype (73.3\%) but all SPMS subjects were less than 40 years old. The summary of brain volume measurement and CCI can be seen in Table 1 .

Correlation between corpus callosum index with brain volumetry and clinical characteristics. The CCI was significantly correlated with CCV ( $\mathrm{r}=0.79$, $p<0.0001)$. Significant correlation was also found between CCI and cortical gray matter, subcortical gray matter and white matter volumes (Figure 2).

The CCI was significantly correlated with age of onset $(\mathrm{r}=0.56, p=0.001)$ and inversely with EDSS $(\mathrm{r}=-0.77, p<0.0001)$. However, the frequency of relapse showed no correlation with CCI. Similar result was also found in correlation between CCV and age of onset, EDSS, and frequency of relapse.

Comparison of CCV and CCI between RRMS and SPMS subtype. The EDSS in SPMS were significantly higher than RRMS subjects $(p=0.003)$. The CCV was smaller in SPMS with $p=0.004$. The CCI was also 
smaller in SPMS with $p=0.008$.

Discussion. The CCI was found to have significant correlation with CCV as well as cortical gray matter, subcortical gray matter, and white matter volumes. The CCI was also positively correlated with age of onset and inversely with EDSS. The EDSS in SPMS was significantly higher than RRMS subjects.

The significant correlation between the CCI and the cortical gray matter volume $(\mathrm{r}=0.64)$ observed in this study showed a diffuse pathological process in the corpus callosum and cortical gray matter of MS patients. Significant reductions in gray matter volume, especially in the frontotemporal cortex, could be found in RRMS patients. This gray matter volume reduction progressively occurred in patients with progressing lesions in their white matter. This finding indicated that cortical gray matter regional atrophy correlated with progressing lesions in the white matter. However, it is still unclear whether the atrophy process in gray matter occurred due to continuous tissue damage in the white matter, such as axonal transection and retrograde neurodegeneration, or if it occurred independently. ${ }^{28}$ Jehna et $\mathrm{al}^{32}$ showed a correlation between the periventricular lesion and cortex depletion, which indicated a pathological process, thus supporting the inflammation factor theory.

A previous study found that CCI was significantly correlated with CCV and white matter volume in RRMS patients ${ }^{23}$ whereas in this study we also sought the similar findings not only in RRMS but also in SPMS patients. CCI also weakly and moderately correlated with brain parenchymal fraction (BPF) and whole brain volume. $^{23,24}$

A significant correlation $(r=0.81)$ between the CCI and cerebral white matter indicated a similar pathological process since both of them are white matter structures. Similarly, Goncalves mentioned a significant correlation between the CCI with white matter and lesion volume. ${ }^{26}$

A pathology study by Evangelou showed that lesion load in the white matter was significantly correlated with corpus callosum axonal density in the neuronal tract projection region. This finding indicated that Wallerian degeneration occurred after axonal transection in axons that passed through the inflamed area. ${ }^{27}$

Comparing to brain volumetry technique, CCI measurement is very easy and fast. Corpus callosum morphometric volume measurement using FreeSurfer@ is a long-standing method that requires approximately 14 hours of computation per subject, compared to 18 seconds using CCI methods. This CCI method also had an inter-rater agreement level of 0.94 and intra-rater agreement level of $0.96 .{ }^{19}$

The present study showed a significant correlation $(\mathrm{r}=0.69)$ between the CCI and subcortical gray matter volume, which could represent diffuse pathological processes that occurred in both structures. Cortical gray matter atrophy occurred in the early phase of MS; however, the subcortical gray matter atrophy occurred earlier than cortical gray matter atrophy. Thalamus atrophy was reported in patients with primary progressive multiple sclerosis (PPMS) and RRMS in the early phase, whereas cortical atrophy occurred in the late phase of MS. Another study reported that MS patients' thalamus volumes decreased by $17 \%$, compared to healthy controls, with a significant correlation between the width of the third ventricle and thalamus atrophy $(\mathrm{r}=-0.59 ; p<0.05)$. Subcortical gray matter atrophy occurred in almost all the MS patients, compared to healthy controls. The longitudinal subcortical gray matter atrophy rate was faster than that of other brain structures in MS patients. ${ }^{28,29}$

The pathological process that affected subcortical gray matter atrophy remained unclear. The subcortical gray matter structure had extensive neural connections with the cortical gray matter; therefore, the subcortical gray matter atrophy was an impact of retrograde and anterograde neurodegeneration that connected both regions. ${ }^{30}$ Koskimaki et $\mathrm{al}^{31}$ mentioned that thalamus atrophy was the result of bidirectional association with damaged cortical structures in MS patients and the location was near the choroid plexus, a place where the immune system enters the central nervous system and therefore would be more subject to inflammation processes. Though the pathophysiology process differed between corpus callosum atrophy and subcortical gray matter atrophy, there was a significant correlation between the CCI and the subcortical gray matter volume due to the diffuse pathological processes that occurred simultaneously. This finding supports the CCI's potential as a marker for describing pathological processes that occurred in the brains of MS patients.

Besides showed correlation between CCI and brain volumetry, our study also found correlation with age of onset and EDSS ( $\mathrm{r}=0.56, p=0.001$ and $\mathrm{r}=-0.77$, $p<0.0001)$. Not all previous studies found similar result as ours. ${ }^{23,26,33}$ The EDSS and disease duration was significantly inverse correlated with CC thickness, fractional anisotropy (FA) and mean diffusivity (MD) in RRMS patients. ${ }^{34}$ Another study evaluated thickness and FA of midsagittal CC in hierarchical clustering algorithm found three subgroups severity of CC 
damage that corresponds with disease duration, clinical disability and cognitive impairment. ${ }^{35}$

A longitudinal study showed that corpus callosum atrophy would be more aggressive in the early phase of MS. The reduction of brain volume rate decreased as the disease duration increased. Another study showed that a corpus callosum atrophy rate of $2 \%$ in the first year could be a long-term predictor of disability enhancement in MS patients. ${ }^{19,36}$

Our study have some limitations, first the sample size is small and the design in retrospective study and the second we did not evaluate the interrater agreement between radiologists in obtaining the CCI value.

In conclusion, the present study found correlations between the CCI and cortical gray matter, subcortical gray matter, and white matter volumes as well as the age of onset. The CCI was also inversely correlated with EDSS. Thus, the current study adds more value of CCI as a surrogate marker for brain atrophy. The easily and fast technique to obtain make the CCI as an option in MS management evaluation particularly in the developing country like Indonesia where MRI with volumetric study yet very limited. Validity of the CCI in clinical setting need to be evaluated for the future research.

Acknowledgment. Thank you to Holy Sarah Gita, Admiral Conollin Zega, and Dhevita Indyana Putri for preparing the manuscript. We also would like to thank American Manuscript Editors (https://www.americanmanuscripteditors.com) for English language editing.

\section{References}

1. Brownlee WJ, Hardy TA, Fazekas F, Miller DH. Diagnosis of multiple sclerosis: progress and challenges. Lancet 2017; 389: 1336-1346.

2. Dendrou CA, Fugger L, Friese MA. Immunopathology of multiple sclerosis. Nat Rev Immunol 2015; 15: 545-558.

3. Howard J, Trevick S, Younger DS. Epidemiology of Multiple Sclerosis. Neurol Clin 2016; 34: 919-939.

4. Garg N, Reddel SW, Miller DH, Chataway J, Riminton DS, Barnett $\mathrm{Y}$, et al. The corpus callosum in the diagnosis of multiple sclerosis and other CNS demyelinating and inflammatory diseases. J Neurol Neurosurg Psychiatry 2015; 86: 1374-1382.

5. Cheong WL, Mohan D, Warren N, Reidpath DD. Multiple Sclerosis in the Asia Pacific Region: A Systematic Review of a Neglected Neurological Disease. Front Neurol 2018; 9.

6. Islas MÁM, Ciampi E. Assessment and impact of cognitive impairment in multiple sclerosis: An overview. Biomedicines 2019; 7: 1-19.

7. Sumowski JF, Benedict R, Enzinger C, Filippi M, Geurts JJ, Hamalainen P, et al. Cognition in multiple sclerosis: State of the field and priorities for the future. Neurology 2018; 90: 278-288.

8. Leavitt VM, Tosto G, Riley CS. Cognitive phenotypes in multiple sclerosis. J Neurol 2018; 265: 562-266.

9. Estiasari R, Fajrina Y, Lastri DN, Melani S, Maharani K,
Imran D, et al. Validity and Reliability of Brief International Cognitive Assessment for Multiple Sclerosis (BICAMS) in Indonesia and the Correlation with Quality of Life. Neurol Res Int 2019; 2019: 1-7.

10. Ghione E, Bergsland N, Dwyer MG, Hagemeier J, Jakimovski D, Paunkoski I, et al. Brain Atrophy Is Associated with Disability Progression in Patients with MS followed in a Clinical Routine. Am J Neuroradiol 2018; 39: 2237-2242.

11. Lassmann H. Multiple sclerosis pathology. Cold Spring Harb Perspect Med 2018; 8: 1-16.

12. Popescu V, Klaver R, Voorn P, Galis-De Graaf Y, Knol DL, Twisk JWR, et al. What drives MRI-measured cortical atrophy in multiple sclerosis? Mult Scler 2015; 21: 1280-1290.

13. Rojas JI, Patrucco L, Miguez J, Cristiano E. Brain atrophy in multiple sclerosis: therapeutic, cognitive and clinical impact. Arq Neuropsiquiatr 2016; 74: 235-243.

14. Grothe M, Lotze M, Langner S, Dressel A. The role of global and regional gray matter volume decrease in multiple sclerosis. J Neurol 2016; 263: 1137-1145.

15. Bergsland N, Horakova D, Dwyer MG, Uher T, Vaneckova M, Tyblova M, et al. Gray matter atrophy patterns in multiple sclerosis: A 10-year source-based morphometry study. NeuroImage Clin 2018; 17: 444-451.

16. Fisher E, Lee JC, Nakamura K, Rudick RA. Gray matter atrophy in multiple sclerosis: A longitudinal study. Ann Neurol 2008; 64: 255-265.

17. Alroughani R, Deleu D, El Salem K, Al-Hashel J, Alexander KJ, Abdelrazek MA, et al. A regional consensus recommendation on brain atrophy as an outcome measure in multiple sclerosis. BMC Neurol 2016; 16: 240.

18. Llufriu S, Blanco Y, Martinez-Heras E, Casanova-Molla J, Gabilondo I, Sepulveda M, et al. Influence of Corpus Callosum Damage on Cognition and Physical Disability in Multiple Sclerosis: A Multimodal Study. Paul F, editor. PLoS One 2012; 7: e37167.

19. Granberg T, Martola J, Bergendal G, Shams S, Damangir S, Aspelin P, et al. Corpus callosum atrophy is strongly associated with cognitive impairment in multiple sclerosis: Results of a 17-year longitudinal study. Mult Scler J 2015; 21: 1151-1158.

20. Papathanasiou A, Messinis L, Zampakis P, Papathanasopoulos P. Corpus callosum atrophy as a marker of clinically meaningful cognitive decline in secondary progressive multiple sclerosis. Impact on employment status. J Clin Neurosci 2017; 43: 170-175.

21. Dewey J, Hana G, Russell T, Price J, McCaffrey D, Harezlak J, et al. Reliability and validity of MRI-based automated volumetry software relative to auto-assisted manual measurement of subcortical structures in HIV-infected patients from a multisite study. Neuroimage 2010; 51: 1334-1344.

22. Vidal-Jordana A, Pareto D, Sastre-Garriga J, Auger C, Ciampi E, Montalban X, et al. Measurement of Cortical Thickness and Volume of Subcortical Structures in Multiple Sclerosis: Agreement between 2D Spin-Echo and 3D MPRAGE T1-Weighted Images. Am J Neuroradiol 2017; 38: 250-256.

23. Figueira FFA, Santos VS dos, Figueira GMA, Silva ÂCM da. Corpus Callosum Index: A practical method for long-term follow-up in multiple sclerosis. Arq Neuropsiquiatr 2007; 65: 931-935.

24. Yaldizli Ö, Atefy R, Gass A, Sturm D, Glassl S, Tettenborn $\mathrm{B}$, et al. Corpus callosum index and long-term disability in multiple sclerosis patients. J Neurol 2010; 257: 1256-1264.

25. Fischl B. FreeSurfer. Neuroimage 2012; 62: 774-781. 
26. Gonçalves LI, dos Passos GR, Conzatti LP, Burger JLP, Tomasi $\mathrm{GH}$, Zandoná MÉ, et al. Correlation between the corpus callosum index and brain atrophy, lesion load, and cognitive dysfunction in multiple sclerosis. Mult Scler Relat Disord 2018; 20: 154-158.

27. Evangelou N. Regional axonal loss in the corpus callosum correlates with cerebral white matter lesion volume and distribution in multiple sclerosis. Brain 2000; 123: 1845-1849.

28. Radü E, Bendfeldt K, Mueller-Lenke N, Magon S, Sprenger T. Brain atrophy: an in-vivo measure of disease activity in multiple sclerosis. Swiss Med Wkly 2013; 143: 1-11.

29. Eshaghi A, Prados F, Brownlee WJ, Altmann DR, Tur C, Cardoso MJ, et al. Deep gray matter volume loss drives disability worsening in multiple sclerosis. Ann Neurol 2018; 83: 210-222.

30. Pukenas B. Normal Brain Anatomy on Magnetic Resonance Imaging. Magn Reson Imaging Clin N Am 2011; 19: 429-437.

31. Koskimäki F, Bernard J, Yong J, Arndt N, Carroll T, Lee SK, et al. Gray matter atrophy in multiple sclerosis despite clinical and lesion stability during natalizumab treatment. Bergsland N, editor. PLoS One 2018; 13: e0209326.
32. Jehna M, Pirpamer L, Khalil M, Fuchs S, Ropele S, Langkammer C, et al. Periventricular lesions correlate with cortical thinning in multiple sclerosis. Ann Neurol 2015; 78: 530-539.

33. Yaldizli Ö, Penner IK, Frontzek K, Naegelin Y, Amann M, Papadopoulou A, et al. The relationship between total and regional corpus callosum atrophy, cognitive impairment and fatigue in multiple sclerosis patients. Mult Scler J 2014; 20: 356-364.

34. Caligiuri ME, Barone S, Cherubini A, Augimeri A, Chiriaco $\mathrm{C}$, Trotta $\mathrm{M}$, et al. The relationship between regional microstructural abnormalities of the corpus callosum and physical and cognitive disability in relapsing-remitting multiple sclerosis. NeuroImage Clin 2015; 7: 28-33.

35. Barone S, Caligiuri ME, Valentino P, Cherubini A, Chiriaco C, Granata A, et al. Multimodal assessment of normal-appearing corpus callosum is a useful marker of disability in relapsingremitting multiple sclerosis: an MRI cluster analysis study. $J$ Neurol 2018; 265: 2243-2250.

36. Vaneckova M, Kalincik T, Krasensky J, Horakova D, Havrdova E, Hrebikova T, et al. Corpus Callosum Atrophy - A Simple Predictor of Multiple Sclerosis Progression: A Longitudinal 9-Year Study. Eur Neurol 2012; 68: 23-27.

Student Corner
We invite students from a variety of medical disciplines to submit original contributions based
on their supervised research.
The Student Corner of Saudi Med J aims to help students explore research opportunities
and network with other peers and mentors in the same field.
Submission Guidelines
Submitted Abstracts should include the following:
- Title should be descriptive
Author's names and affiliation(specify college level/year, academic degree of Senior Author)
Abstract must be structured and not more than 300 words
The following are the typical headings:
Objectives (background, why the study was done, specific aims)
Methods (setting, date of study, design, subjects, intervention and analysis)
Results (findings, data and statistical tests) and
Conclusion (general interpretation of results)
General Information on Abstract Submission
Submitted Abstracts should be co-authored by a Senior Supervisor
Abstracts will be reviewed by Student's Corner Section Editor
There is no fee to submit an Abstract
Ethical Approval should be provided
Non-indexed materials

